\title{
Should patients with sleep apnoea/hypopnoea syndrome be diagnosed and managed on the basis of home sleep studies?
}

\author{
O. Parra*, N. García-Esclasans*, J.M. Montserrat**, L. García Eroles*, \\ J. Ruíz*, J.A. López*, J.M. Guerra*, J.J. Sopeña*
}

\begin{abstract}
Should patients with sleep apnoealhypopnoea syndrome be diagnosed and managed on the basis of home sleep studies? O. Parra, N. García-Esclasans, J.M. Montserrat, L. García Eroles, J. Ruíz, J.A. López, J.M. Guerra, J.J. Sopeña. @ERS Journals Ltd 1997.

ABSTRACT: The purpose of this study was to analyse the validity and the economic efficiency of a portable monitor of respiratory parameters (PMRP), used in a home setting for the diagnosis of sleep apnoea/hypopnoea syndrome (SAHS).

Eighty nine patients with suspected SAHS were studied in two settings: in the sleep laboratory using full-polysomnography (full-PSG); and at the patient's home using a PMRP. In the home setting, 50 patients were assisted by a technician and 39 set up the equipment themselves. SAHS (apnoea/hypopnoea index (AHI) $>10$ events $\cdot h^{-1}$ by means of full-PSG) was diagnosed in 75 of the 89 patients. An acceptable agreement was obtained between the AHI measured by full-PSG and PMRP, according to the Bland and Altman method of concordance (mean bias $2.56 ; 95 \%$ confidence interval 3.25). Sensitivity and specificity of PMRP were adequate for diagnostic purposes; however, their values rely on the prior PMRP-AHI cut-off point selected with reference to full-PSG-AHI $>10$. The clinical therapeutic decision taken after PMRP agreed with that taken with full-PSG in 79 patients $(89 \%)$. Although $10 \%$ of the studies with an individual set-up needed repetition, both of the domiciliary modalities (with and without a technician's intervention) were, economically, about three times more efficient than full-PSG.

In conclusion, we believe that patients with a suspected sleep apnoea/hypopnoea syndrome should initially be studied in a home setting with a portable monitor of respiratory parameters, since it is a reliable method with an acceptable cost-effective profile.
\end{abstract}

Eur Respir J 1997; 10: 1720-1724.

\begin{abstract}
* Servei de Pneumologia, Hospitals de Barcelona (Quinta Salut Aliança), Barcelona, Spain. **Hospital Clínic, Universitat de Barcelona, Spain.
\end{abstract}

Correspondence: O. Parra

Servei de Pneumologia

C/Pare Claret 200

08025 Barcelona

Spain

Keywords: Cost-effective

domiciliary diagnosis

sleep apnoea/hypopnoea index

Received: April 111996

Accepted after revision January 201997

Supported by Grants 94/1006 from Fondo de Investigaciones Sanitarias de la Seguridad Social (FIS).
Sleep apnoea/hypopnoea syndrome (SAHS) is a disorder with a high prevalence [1]. Associated morbidity and mortality [2-5] are increased, but effective treatment exists for symptomatic patients [2,6]. Full-polysomnography (full-PSG), which is an attended recording of neurological and respiratory parameters, is accepted as the gold standard for SAHS diagnosis. However, this standard raises two major problems: firstly, its lack of accessibility, because it is limited to specialized centres; and, secondly, its high cost. High prevalence, accessibility and cost problems are the main reasons that justify research into more available and less costly, but comparably reliable, alternatives. A recent study has shown that accurate diagnosis can also be made only on the basis of night-time respiratory recordings [7]. Recently, we have been able to use a partially attended system at an ordinary hospital ward, with acceptable results [8].

Similar systems have been conceived as portable equipment, which makes them suitable for a home setting [9]. As additional advantages, simplified home equipment may favour a more physiological sleep and probably a lower cost. However, drawbacks from home studies have been noted due to the possible unsatisfactory results obtained because of faulty placing of sensors or poor quality signals. Both are potential disadvantages of an unattended method, and could lead to the need for repetition of studies, with consequent increase in cost, so that the procedure is no longer cost-effective.

Therefore, the purpose of this study was to validate the use of a portable monitor of respiratory parameters (PMRP) in a home setting, as a method for diagnosis and treatment in comparison with full-PSG. The efficacy and the cost of such a diagnostic method is also provided.

\section{Methods}

\section{Patients}

The study population consisted of consecutive patients referred to our hospital for evaluation of suspected SAHS. A physician experienced in the diagnosis and management of patients with sleep disorders, interviewed (using 
a clinical questionnaire) and examined the patients, and decided to perform a sleep study in 89 cases. Within a 1 month period, diagnostic sleep studies were performed at random in two settings: 1) in a sleep laboratory using full-PSG; and 2) at the patient's home using a PMRP, with a technologist's intervention in the set-up of the equipment in 50 cases, and with the patient's own setup in the remaining 39 . The latter group received written instructions, as well as a 10 min period of training provided by a technician, who used a dummy to teach them. In this case, the domiciliary study always preceded full-PSG to avoid the influence of training.

\section{Overnight full-PSG}

This included electroencephalogram (C4/A1, C3/A2, O2/A1, O1/A2), chin electromyogram and electro-oculogram recordings for sleep staging, according to the established standard criteria [10]. Arterial oxygen saturation $\left(\mathrm{Sa}, \mathrm{O}_{2}\right)$ was measured continuously with a finger probe using a pulse oximeter (504 Critical Care System Inc., Waukesha, WI, USA). Ribcage and abdominal motion were monitored by piezoelectric bands placed over the thorax and abdomen (Resp-Ez., Tm Bionic, Mid-lothian, VA, USA). Airflow was assessed using a thermistor. All signals were recorded continuously through a polygraph (Nicolet 1A98; Madison, WI, USA). Respiratory events were scored as apnoeas when there was a cessation of airflow lasting $\geq 10 \mathrm{~s}$, and hypopnoeas when any discernible reduction of airflow or period of thoraco-abdominal paradoxical motion lasting $\geq 10 \mathrm{~s}$ was observed, associated with an arousal or with a cyclical dip in $\mathrm{Sa}_{2} \mathrm{O}_{2}$. Arousals were defined according to the scoring rules of the American Sleep Disorders Association [11]. An apnoea/hypopnoea index (AHI) $>10$ events $\cdot \mathrm{h}^{-1}$ was considered diagnostic of SAHS.

\section{Portable respiratory recordings in home setting}

Domiciliary studies were performed using a portable device that measures nasal/oral airflow (thermistry), chest wall impedance, oxygen saturation, heart rate (finger pulse oximetry), snoring and body position (Edentec Monitoring System, Model 3711 Edentrace II, MN, USA). The morning after the study, the analogue signals were digitized and displayed on the computer screen, allowing a visual assessment of the overnight tracings. Respiratory events were scored manually using similar criteria to those used for full-PSG. The definition for apnoea was exactly the same, but hypopnoea was considered as any reduction in airflow or thoracic motion lasting $>10 \mathrm{~s}$, associated with any cyclical dip of $\mathrm{Sa}, \mathrm{O}_{2}$ $\geq 2 \%$. AHI was calculated taking into account the time spent in bed.

\section{Economic analysis}

The cost of domiciliary studies was assessed by systematic recording and classification of expenditures, including mainly single-use material and the technologist's time, in two ways: 1) with the technologist's intervention in the setting-up of the equipment (50 cases, including the travel time and interpretation of the recordings); and 2) without the technologist's intervention (39 patients, including the technician's time to instruct the patient, and the interpretation time). We consider effectiveness to be the number of valid studies. False-negatives or unachieved studies that would have required a full-PSG, and those with inaccurate signals or inadequate recordings for interpretation, that would have required a repetition of PMRP, were considered as not effective. Their cost (either because of repetition of PMRP or need for full-PSG) was added proportionally to the cost-per-unit of PMRP. The cost of establishing a new diagnostic method was also considered. We considered an output of 200 studies per year and a linear depreciation of the equipment in 5 yrs time. The cost of full-PSG in our unit has been calculated at Pts 52,477.7 (US \$433.7).

\section{Data analysis}

Data are expressed as mean (SD). The same technician carried out the analysis both of full-PSG and PMRP, being unaware of the patients' identities. Results of the first test were not available until the second test was performed. The mean AHI obtained with PMRP was compared to that obtained with full-PSG, using a paired t-test. The results both of full-PSG and PMRP were compared in three different ways: 1) agreement between AHI obtained from PMRP (PMRP-AHI) and full-PSG (full-PSG-AHI) was analysed according to the BLAND and Altman [12] method of concordance; 2) evaluation of the diagnostic usefulness in terms of sensitivity and specificity, obtaining a receiver operating characteristic (ROC) curve by taking different PMRP-AHI cut-off points with respect to full-PSG-AHI $>10$; and, finally, 3 ) independent comparison of the clinical therapeutic decision outcome both through the clinical findings and the AHI obtained from full-PSG versus PMRP. In our unit, the decision to treat with nasal continuous positive airway pressure (nCPAP) was made on the basis of the following criteria: 1) documented SAHS (AHI >10) in the presence of clear clinical impairment [13]; and 2 ) an AHI >30, even with moderate clinical impairment.

\section{Results}

\section{Full-PSG and PMRP results}

The mean (SD) age of the 89 patients $(73$ males and 16 females) studied was 54 (12) yrs, with a mean body mass index of $29(4) \mathrm{kg} \cdot \mathrm{m}^{-2}$. After the performance of full-PSG, 75 of the 89 patients $(84 \%)$ were diagnosed as having SAHS (>10 events. $\mathrm{h}^{-1}$ ); whereas, after the performance of a PMRP, 73 of the 89 patients $(82 \%)$ were diagnosed as having SAHS $\left(>10\right.$ events $\left.\cdot \mathrm{h}^{-1}\right)$. The mean AHI obtained by full-PSG and PMRP was not significantly different (34.3 (25) and 31.8 (27), respectively). The mean number of apnoeas $\cdot \mathrm{h}^{-1}$ (apnoea index (AI)) obtained using full-PSG and PMRP was 8.7 (13.9) and $10.9(16)$, respectively ( $\mathrm{p}<0.05)$; whereas, the mean number of hypopnoeas $\cdot \mathrm{h}^{-1}$ (hypopnoea index (HI)) obtained using full-PSG and PMRP was 25.5 (19.7) and 20.5 (14.2), respectively $(\mathrm{p}<0.05)$. 


\section{Agreement in AHI measurement}

The agreement in AHI between full-PSG and PMRP, using the method suggested by Bland and Altman [12], showed the following results: the mean bias was 2.56, with a $95 \%$ confidence interval $(95 \% \mathrm{CI})$ of 3.25 (not significantly different from zero) (fig. 1).

\section{Diagnostic value of PMRP}

Figure 2 shows a ROC curve plotting sensitivity and specificity of the domiciliary method at different cutoff points for AHI obtained by the PMRP (PMRP-AHI), with respect to full-PSG-AHI $>10$. It can be seen that the sensitivity and specificity vary depending on the chosen PMRP-AHI cut-off point. A PMRP-AHI cut-off point of 18 showed a sensitivity of $73 \%$ and a specificity of $80 \%$. If the PMRP-AHI cut-off point is reduced to 8 , the sensitivity rises to $95 \%$, but specificity goes down to $33 \%$. If the cut-off point is increased to 23 , a specificity of $93 \%$ is achieved, but sensitivity decreases to $63 \%$. Therefore, if an AHI below 8 is obtained, it can be assumed that the probability of having SAHS is very low, whereas if an AHI above 23 is obtained, the probability of having SAHS is high. When an AHI between 8 and 23 is obtained, there is a chance of having false-negative and/or false-positive results.

\section{Analysis of the clinical therapeutic decision}

The clinical therapeutic decision after PMRP agreed with that from full-PSG in 79 patients (89\%). Only in 10 patients was there a real disagreement in the decision to treat with nCPAP: six patients treated with nCPAP according to full-PSG would not be treated according to PMRP, and the reverse would have occurred in four cases. In the group of six undertreated patients according to PMRP, four had a PMRP-AHI >10 and were cor-

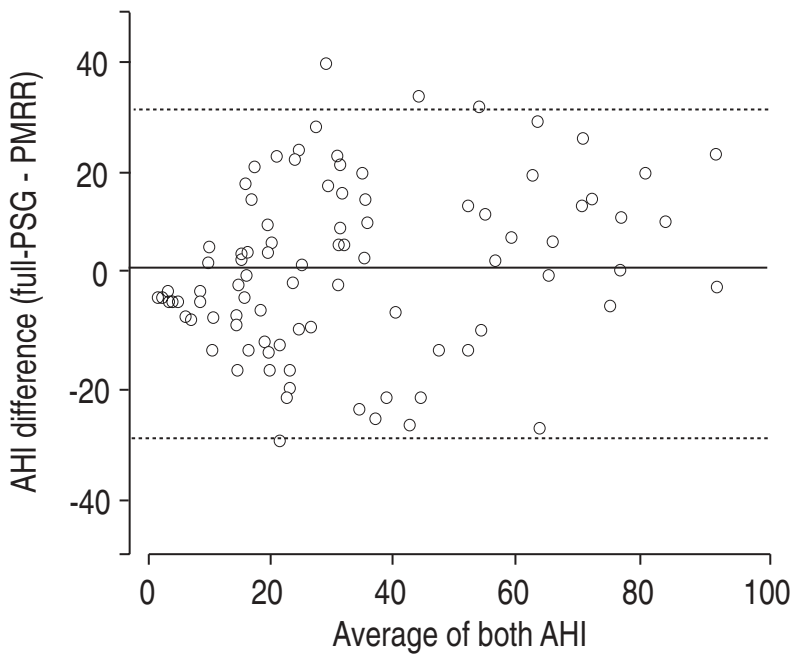

Fig. 1. - Comparison of AHI measured by the two methods (fullPSG vs PMRP). The solid line represents the mean bias (2.56) of the individual differences and the dotted lines are the limits of agreement (mean bias $\pm 2 \mathrm{SD}$ of the differences). AHI: apnoea/hypopnoea index; full-PSG: full polysomnography; PMRP: portable monitor of respiratory parameters.

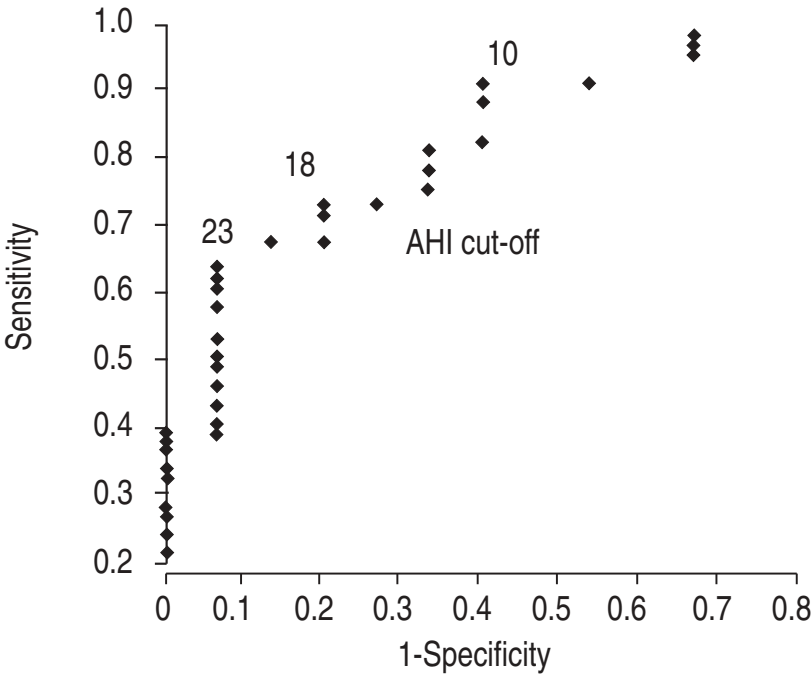

Fig. 2. - Receiver operating characteristics (ROCs) for different PMRP-AHI cut-off points, referred to a full PSG-AHI $>10$. Each point represents sensitivity plotted against specificity corresponding to different cut-off points of PMRP-AHI. For example, for an AHI of 18, a sensitivity of $73 \%$ and a specificity of $80 \%$ was obtained. As a lower cut-off point for AHI was chosen, a better sensitivity and a worse specificity was obtained, and vice versa, as a higher cut-off point for AHI was chosen.

rectly diagnosed as having SAHS, but the PMRP-AHI was $<30$ and few symptoms were present. In the four overtreated patients according to PMRP, the PMRP-AHI was >30; SAHS was correctly diagnosed in all, in that the full-PSG-AHI was $>10$ but $<30$.

\section{Economic analysis}

Table 1 includes the calculated direct and indirect costs for the two different PMRP diagnostic modalities (with or without a technologist's intervention). We have assumed a linear annual depreciation value of the PMRP device of Pts 341,994.4 (US\$2,826.4). Effectiveness of PMRP with the technologist's intervention was $92 \%$, since we considered that there were $8 \%$ false-negatives (taking an AHI cut-off point of 10). Effectiveness of PMRP without a technologist's intervention was

Table 1. - Classification of PMRP costs

\begin{tabular}{|c|c|c|c|c|}
\hline \multirow[t]{2}{*}{ Costs } & \multicolumn{2}{|c|}{$\begin{array}{l}\text { PMRP with } \\
\text { technician }\end{array}$} & \multicolumn{2}{|c|}{$\begin{array}{l}\text { PMRP without } \\
\text { technician }\end{array}$} \\
\hline & Pts & (US\$) & & (US\$) \\
\hline \multicolumn{5}{|l|}{ Direct cost } \\
\hline Single-use & 6861 & $(56.7)$ & 6861 & (56.7) \\
\hline Technician & 5094 & $(42.1)$ & 1694 & (14.0) \\
\hline False-negatives & 4090 & $(33.8)$ & 4090 & (33.8) \\
\hline Establishing new method & 290 & $(2.4)$ & 290 & (2.4) \\
\hline Repetition of studies & & & 1646 & (13.6) \\
\hline Unachievable studies & & & 1041 & $(8.6)$ \\
\hline \multicolumn{5}{|l|}{ Indirect cost } \\
\hline Repairs & 641 & $(5.3)$ & 641 & (5.3) \\
\hline Travel & 992 & $(8.2)$ & 992 & $(8.2)$ \\
\hline Total & 17968 & $(148.5)$ & 17255 & (142.6) \\
\hline Annual depreciation" & 341994 & (2826.4) & & \\
\hline
\end{tabular}

\#: based on 200 studies per year. PMRP: portable monitor of respiratory parameters; Pts: Spanish pesetas; US\$: United States dollars. 
$80 \%$, since we considered that there were $8 \%$ false-negatives, $2 \%$ unachievable, and $10 \%$ faulty studies that required a repetition. The total cost was Pts $17,968.5$ (US\$148.5) for PMRP with a technologist's intervention and Pts 17,254.6 (US\$142.6) without. If we consider the actual cost of a full-PSG in Spain (Pts 52,477.7 (US\$433.7)), PMRP, either with or without a technologist's intervention, is three times more efficient than PSG, since the cost of a full-PSG is equivalent to the cost of three effective PMRP procedures.

\section{Discussion}

This study shows that domiciliary use of a portable respiratory recording device can be an effective and less costly way to assess SAHS diagnosis. The acceptable agreement between PMRP-AHI and full-PSG-AHI makes the former a proper method, despite its trend to underestimate hypopnoeas. The diagnostic usefulness of the domiciliary method is supported by its acceptable sensitivity and specificity. Furthermore, in most cases, the clinical decision to treat based on the PMRP results is in agreement with that from full PSG.

Only a few studies evaluating the application of portable recordings have been published. Furthermore, most of the equipment has been tested in a laboratory setting with simultaneous attended full-PSG monitoring, rather than in the home setting [9, 14-19]. Only two of these studies have attempted to use such equipment at the patient's home $[9,11]$. In the work of ANCOLI-ISRAEL et al. [15], the equipment used was different from that used in the present study. REDLINE et al. [9] used a device (Edentrace 4700) similar to that used in the present study, but only a small number of patients were studied at home. However, they found a good correlation in respiratory disturbance between portable recordings and full-PSG, and good reproducibility of the method.

Despite the agreement obtained applying the BLAND and Altman [12] method of analysis, we think that a more realistic approach to PMRP diagnostic usefulness can be reached by analysing the relationships between sensitivity and specificity, and, moreover, by comparing the clinical therapeutic decision on the patient's outcome depending on the procedure considered, rather than based on the AHI itself. With regard to the ROC curves obtained, different sensitivity and specificity values can be achieved depending on the PMRP-AHI cutoff point selected. When the values of PMRP-AHI are $8-23$, false-negative or false-positive results are possible, but the therapeutic decision on these patients will not be very different, since their SAHS will not be severe. The main reason for the limited sensitivity of the test is the underestimation of AHI, due to underrecognition of hypopnoeas (paradoxical thoracoabdominal motion and movement arousals cannot be detected with PMRP), and to the fact that AHI obtained by full-PSG is calculated taking into account the actual sleep time, whereas in the PMRP it is calculated taking into account the time spent in bed. Differences in posture and nightto-night AHI variability may have had an influence on the discrepancies observed, and should be considered, especially at low AHI values [20]. Although this variability can also influence specificity, we could specu- late that the main factor that restricts specificity could be the better sleep efficiency at the patient's home, compared to a night spent in the strange surroundings of a sleep laboratory, which can modify sleep architecture and efficiency. This fact raises the controversial question of how effective full-PSG is as a gold standard for the diagnosis of SAHS.

When analysing the clinical therapeutic decision based on PMRP-AHI or full-PSG-AHI plus the presence of clinical impairment [13], we reach an $89 \%$ complete agreement. Discordance occurred in only 10 patients out of $89(11 \%)$. The main reason for this discrepancy was the decision to treat patients with an AHI $>30$ in spite of the few clinical symptoms.

We are not aware of any published work analysing the cost of each test in domiciliary studies. We have calculated it thoroughly for the two different modalities, with and without a technologist's intervention in the setting-up of equipment. Admission to a specialized hospital and the presence of technologists during the night account for the major part of full-PSG costs. PMRP used in a home setting omits these elements, but additional costs should be included, such as the technologist's travel time when present, and the need for multiple and repetitive studies to diagnose one patient. The effectiveness reached for PMRP with a technologist is very good, up to $92 \%$ of valid studies. Here we have not considered false-positives as invalid studies, since we think they are actually positive studies, not detected by fullPSG. However, night-to-night variability or some recording artefacts could not be fully excluded. In the modality without a technologist, $10 \%$ of studies had to be repeated, with a consequent increase in cost, thus explaining the slight cost difference between the two modalities. Even so, in both cases, the cost is about three times lower than that calculated for full-PSG, considering that these amounts could be different within countries. We should expect a further reduction in cost after identifying the most common errors, and by changing the single-use material for multiple-use sensors. Finally, we can state that PMRP, with or without a technologist, is economically efficient. Although the transformation of an "in-patient service" into a "home health service" is not always cost-effective, we can speculate that this could be the case.

In recent years, several approaches have been developed to diagnose and treat sleep apnoea in an easier and cheaper way than full overnight PSG, such as nap studies or split night for diagnosis and nCPAP treatment [21], but, on the whole they are satisfactory only in severe cases. Although domiciliary diagnosis of SAHS would need a second study for nCPAP titration when indicated, it is easier, cheaper and more broadly available than the conventional approach. Nevertheless, we believe that the use of such simplified systems should be performed in centres with access to full-PSG, to provide an adequate yield.

In conclusion, the portable monitor of respiratory parameters used in a home setting can be a very important support to the routine practice of a sleep laboratory. It provides a confident diagnosis of sleep apnoea/hypopnoea syndrome and a correct therapeutic outcome decision in most cases. In addition, it offers advantages compared to full-polysomnography, being more accessible 
and less costly. General implementation of these devices should greatly reduce the number of full-polysomnography studies required. We suggest that patients suspected of suffering sleep apnoea/hypopnoea syndrome should, initially, be studied at home, and that fullpolysomnography should be performed whenever there are diagnostic doubts or when associated conditions are suspected.

\section{References}

1. Young T, Palta M, Dempsey J, Skatrud J, Weber S, Badr S. The occurrence of sleep-disordered breathing among middle-aged adults. $N$ Engl J Med 1993; 328: 1230-1235.

2. He J, Kryger MH, Zorick FJ, Conway W, Roth T. Mortality and apnea index in obstructive sleep apnea: experience in 358 male patients. Chest 1988; 94: 9-14.

3. Partinen M, Guilleminault C. Mortality of patients with obstructive sleep apnea syndrome: a follow up study. Chest 1988; 94: 1200-1204.

4. Koskenvuo M, Kapiro J, Partinen M, Langinvainio H, Sarna S, Heikkila K. Snoring as a risk factor for hypertension and angina pectoris. Lancet 1985; i: 393-396.

5. Hung J, Whitford EG, Parson RW, Hillman DR. Association of sleep apnea with myocardial infarction in men. Lancet 1990; 336: 261-264.

6. Sullivan CE, Issa FG, Berthon-Jones M, Eves L. Reversal of obstructive sleep apnea by continuous positive airway pressure applied through the nares. Lancet 1981; i: 862-865.

7. Douglas NJ, Thomas S, Jan MA. Clinical value of polysomnography. Lancet 1992; 339: 347-350.

8. Carrasco O, Montserrat JM, Lloberes P, et al. Visual and different automatic scoring profiles of respiratory variables in the diagnosis of sleep apnea/hypopnea syndrome. Eur Respir J 1996; 9: 125-130.

9. Redline S, Tosteson T, Boucher MA, Millman R. Measurement of sleep-related breathing disturbance in epidemiologic studies: assessment of the validity and reproducibility of a portable monitoring device. Chest 1991; 100: 1281-1286.
10. Rechtschafen A, Kales A, eds. A manual of standardized terminology, techniques and scoring system for sleep stages of human subjects. Washington, DC, Public Health Service, US Government Printing Office, 1963.

11. ASDA Report. EEG arousals: scoring rules and examples. Sleep 1992; 15: 173-184.

12. Bland JM, Altman D. Statistical methods for assessing agreement between two methods of clinical measurements. Lancet 1986; 1: 307-310.

13. American Thoracic Society. Indications and standards for use of nasal continuous positive airway pressure (CPAP) in sleep apnea syndromes. Am J Respir Crit Care Med 1994; 150: 1738-1745.

14. Emesellem HA, Corson WA, Rappaport BA, Hackett S, Smith LG, Hausfeld JN. Verification of sleep apnea using a portable sleep apnea screening device. South Med J 1990; 83: 748-752.

15. Ancoli-Israel S, Kripke DF, Mason W, Messin S. Comparisons of home sleep recordings and polysomnograms in older adults with sleep disorders. Sleep 1981; 4: 283-291.

16. Gyulay S, Gould D, Sawyer B, Pond D, Mant A, Saunders N. Evaluation of a microprocessor-based portable home monitoring system to measure breathing during sleep. Sleep 1987; 10: 130-142.

17. Svanborg E, Larsson H, Carlsson-Nordlander B, Pirskanen R. A limited diagnostic investigation of sleep apnea syndrome: oximetry and static charge sensitive bed. Chest 1990; 98: 341-345.

18. Salmi TT, Telakivit T, Partinen M. Evaluation of automated analysis of SCSB, airflow, and oxygen saturation signals in patients with sleep related apneas. Chest 1989; 96: 255-261.

19. Stoohs R, Guilleminault C. MESAM 4: an ambulatory device for the detection of patients at risk for obstructive sleep apnea syndrome (OSAS). Chest 1992; 101: $1221-1227$

20. Wittig RM, Romaker A, Zorick FJ, Roehrs TA, Conway WA, Roth T. Night-to-night consistency of apneas during sleep. Am Rev Respir Dis 1984; 129: 244-246.

21. Sanders MH, Kern NB, Constantino JP, et al. Adequacy of prescribing positive airway pressure therapy by mask for sleep apnea on the basis of partial-night trial. Am Rev Respir Dis 1993; 147: 1169-1174. 